\title{
Increase Alginate-Chitosan Nanoparticles Transport Efficiency Through the Epithelium by Attaching nt-PE onto Surface
}

\author{
Ruiying Li, Paul De Bank, Randy Mrsny \\ Department of Pharmacy and Pharmacolody, University of Bath \\ Claverton Down, Bath, BA2 3AN, United Kingdom \\ lr397@bath.ac.uk
}

\section{Extended Abstract}

Nanotechnology has brought drug delivery system into a brand new age---the appearance of Nano delivery system. It has been widely explored in different therapeutic areas. For oral protein delivery, the application of Nano delivery system is limited by the low transport efficiency through the epithelium in the small intestine. The efficient transport of nano delivery system through epithelium requires optimized surface characteristics and specific transport pathway. In this study, chitosan and alginate are chosen for making nanoparticles, as they are bioadhensive, biodegradable and can be modified for the surface modification. The pathway of nanoparticles go across the epithelium is designed to mimic the pathway of virus invasion in the body. Study has shown non-toxic form of pseudomonas exotoxin (nt-PE) can go across the polarized cells (epithelial cells) [1]. Our hypothesis is that the transport efficiency of alginate-chitosan nanoparticles through the epithelium can be increased after attaching nt-PE onto the surface.

Alginate-chitosan nanoparticles were made by ion gelation, the particle size are in the size range of $210 \pm 18 \mathrm{~nm}$ and the zeta potential is $-7 \pm 3 \mathrm{mV}$. After attaching nt-PE onto nanoparticle surface, nanoparticles are in the size range of $192 \pm 17 \mathrm{~nm}$, and the zeta potential is $-10 \pm 4 \mathrm{mV}$. Nt-PE decorated nanoparticles are still in the spherical shape as indicated under Transmission Electron Microscope. This nano-delivery system was tested on Caco-2 cells, an in vitro model of the human intestinal epithelium. The transport efficiency of nt-PE modified nanoparticles are 2 fold more than the unmodified nanoparticles. Nt-PE modified nanoparticles have shown the potential to go across the epithelium. The in vivo transport study is undergoing.

\section{References}

[1] R. J. Mrsny, A. L. Daugherty, et al., "Bacterial toxins as tools for mucosal vaccination,” Drug Discov. Today, vol. 7, no. 4, pp. 247-258, 2002. 